\title{
Alteration in Oral Flora and Effect of Mucositis in Head and Neck Cancer Patients Undergoing Chemo-radiotherapy
}

\author{
Reeta Maurya ${ }^{1,2}$, Manodeep Sen ${ }^{2 *}$ (D) Madhup Rastogi $^{3}\left(\mathbb{D}\right.$ and Somali Sanyal ${ }^{1}$ (iD \\ ${ }^{1}$ Amity Institute of Biotechnology, Amity University, Lucknow - 227 105, Uttar Pradesh, India. \\ ${ }^{2}$ Department of Microbiology, Dr. Ram Manohar Lohia Institute of Medical Sciences, Lucknow - 226 010, Uttar \\ Pradesh, India. \\ ${ }^{3}$ Department of Radiation Oncology, Dr. Ram Manohar Lohia Institute of Medical Sciences, Lucknow - 226010 , \\ Uttar Pradesh, India.
}

\begin{abstract}
The main aim of this study is to determine the various types of oral bacteria and yeast. Present in oral flora of head and neck cancer patients at different stages of chemo-radiotherapy, and compare it with the control group (patients with contralateral healthy mucosa). Seventy seven patients with proven history of squamous cell carcinoma were included in the study. The oral mucosa profile was assessed for bacterial manifestations in swab samples from both the sites of the patients. The grade of mucositis was charted out for all patients during the second and third week of radiotherapy. The study revealed that all isolated oral flora showed a non-significant increase during radiotherapy, while there was a decrease in oral flora in post RT. However, E. faecalis showed a non- significant decrease during RT, while Citrobacter showed an increase. Candida albicans showed $83 \%$ non- significant decrease postradiotherapy. When these floras were correlated with grade of mucositis, an insignificant increase in flora was found in G2 and G3 stage of mucositis. In this study, the effect of radiation was evaluated on oral flora of head and neck cancer patients and compared with contralateral healthy mucosa of the patients. Various changes were observed during and after radiation therapy. In patients with head and neck cancer the normal oral flora are replaced by pathogenic flora during radiotherapy, and the latter is responsible for infections in post- radiation phase.
\end{abstract}

Keywords: Oral flora, Head and neck cancer, Mucositis, Radiotherapy

*Correspondence: sen_manodeep6@yahoo.com; +91 9839446858

(Received: April 17, 2020; accepted: September 18, 2020)

Citation: Maurya R, Sen M, Rastogi M, Sanyal S. Alteration in Oral Flora and Effect of Mucositis in Head and Neck Cancer Patients Undergoing Chemo-radiotherapy. J Pure App/ Microbiol. 2020;14(3):2129-2135. doi: 10.22207/JPAM.14.3.53

(C) The Author(s) 2020. Open Access. This article is distributed under the terms of the Creative Commons Attribution 4.0 International License which permits unrestricted use, sharing, distribution, and reproduction in any medium, provided you give appropriate credit to the original author(s) and the source, provide a link to the Creative Commons license, and indicate if changes were made. 


\section{INTRODUCTION}

Head and neck cancer is one of the highly common cancers in India with higher incidence in men than in women. According to Globocon report published in 2018, head and neck cancer accounts for $16.1 \%$ of all the cases of cancer in men, and when these patients are treated with radiotherapy (RT) or chemotherapy, oral mucositis may develop. Oral mucositis is observed in more than $80 \%$ of RTtreated patients and these side effects can last for more than 5 weeks ${ }^{1}$. Oral squamous cell carcinoma (OSCC) is most commonly observed in oral and oropharyngeal cancer (>90\%). It is reported in literature that about $40 \%$ of patients undergoing chemo-radiotherapy develop oral mucositis ${ }^{2}$. This is also referred to as oral erythematous and ulcerative lesions of various gradings. The degree of mucositis is graded according to guidelines provided by Radiation Therapy Oncology Group (RTOG) and $\mathrm{WHO}^{3}$. It onsets after 5-10 days of commencement of radiotherapy. The grading of mucositis (grade -0, I, II, III, IV) increases with the increase in dose delivered and lasts for 7-14 days after the course of radiotherapy.

Several workers have reported that Candida albicans is the most commonly observed bacterial infection which results in various grades of oral candidiasis (in $60-80 \%$ of cases) in the patients receiving radiotherapy for head and neck cancer $^{4-6}$.

This aim of this study is to evaluate the various types of oral bacteria and yeast. Present in patients with head and neck cancer and evaluate the effect of chemo-radiotherapy on contralateral healthy mucosa.

\section{MATERIALS AND METHODS}

This study was conducted in the departments of Microbiology and Radiation Oncology, Dr. Ram Manohar Lohia Institute of Medical Sciences, Lucknow (a tertiary cancer care institute) from July 2017 to June 2019. Patients included in the study were above 18 years, with histopathologically proven head and neck squamous cell carcinoma including oral cavity, oropharynx, hypopharynx, and larynx) in chemoradiotherapy naive patients who gave informed written consent to participate in the study. Patients having the recurrence of head and neck cancer were not included in the study.
Seventy seven patients (69 males and 8 females) of clinically and histopathologically diagnosed head and neck cancer undergoing chemo-radiotherapy were included in this study. The mean age of the patients was 54 years (range from 30 to 80 years, the majority were in their 50 's). Sample from the healthy contralateral site of the same patients of head and neck cancer served as control Only 19 patients were found suitable to serve as control.

Samples from oral mucosa were collected as per protocol using sterile swabs with identification tags for each patient for bacterial examination. Samples were collected before commencement of radiotherapy, during treatment (after 2 weeks of start of therapy) and after completion of radiotherapy (after 5-6 weeks). For evaluation of control group, oral samples in sterile swabs were collected from the healthy contralateral site of the same patients. One hour prior to swab collection, the patients were not allowed to eat, drink, smoke or use oral hygiene products as per the protocol of proper sample collection.

Swabs were transported to microbiology lab within 2 hours at room temperature for examination. Oral swab samples were spread on MacConkey Agar and blood agar plate (HI Media/ Oxoid Thermo Fisher Scientific). The culture plates were prepared as per standard protocol and incubated under aerobic conditions at $37^{\circ} \mathrm{C}$ for one to two days. For differentiating the Staphylococcus and Enterococcus in these oral samples thus collected, DNase Agar and Arabinose agar base were used.

Using standard departmental protocol, isolated bacterial strains were subjected to various biochemical tests namely, Urease, citrate, methyl red, indole and sulfur indole motility which were specific for oral bacteria such as Lactose fermenter and non- lactose fermenter. Thus these evaluations enabled the identification of the oral bacteria and yeast flora in the study subjects. The other biochemical tests used in this experiment were: Gram staining, catalase test, oxidase test, hemolysis in blood agar plate (BAP), growth in bile aesculin agar.

\section{Grades of Mucositis}

WHO (World Health Organisation) has described the guidelines for grading of radiation 
reaction and mucositis in patients receiving radiotherapy. WHO -oral mucositis assessment criteria is as follows: Grade $0=$ No mucositis; Grade 1= Soreness and Erythema; Grade 2 =Erythema, ulcers, can swallow solid food; Grade 3=Extensive erythematous ulcers, and cannot swallow solid food and Grade 4 = Mucositis to the extent that alimentation is not possible.

Oral mucositis occurred due to side effects of radiation $s$ in the orpharynx (buccal mucosa, tongue and pharynx) were examined. The sample collection was done accordingly. The sites evaluated were: buccal mucosa (left and right), soft and hard palates, border of the tongue (left and right), dorsum of the tongue and the floor of the mouth.

\section{Approval of Ethics Committee}

This is an observational analytical casecontrol study and was duly approved by the Institutional Ethics Committee, Dr. Ram Manohar Lohia Institute of Medical Sciences, Lucknow (IEC No. 28/17).

\section{Statistical analysis}

In this study, the data generated was subjected to statistical analysis with the help of an appropriate bio-statistical tool (SPSS 21.0 for Windows) for interpretation of significant outcome. P-value was used to know the significant outcome considering statistically significant at P-value $<0.05$. P-value was calculated by Fisher's exact test.

\section{OBSERVATIONS AND RESULTS}

The study included 77 patients of histologically proven squamous cell carcinoma undergoing chemo-radiotherapy. Swab samples were obtained from those patients who had healthy contralateral mucosa, and they served as control group and comprised 19 patients (in the ratio of $4: 1$ ). Percentage of males was 89.6 while that of females was 11.3 in the study group. [Table.1]

The diagnosis included $30 \%$ cancers of the oropharynx (23/77), 23\% base of tongue (18/77), $15.5 \%$ of larynx (12/77), $2.5 \%$ of lateral border of tongue $(2 / 77), 2.6 \%$ of tongue $(2 / 77), 9 \%$ of soft palate (7/77), $5 \%$ of pharynx (4/77), $5 \%$ of buccal mucosa (4/77), $5 \%$ of upper alveolar region(4/77) and $1.2 \%$ of throat (1/77) [Table.2]

Treatment comprised a dose of 66.0 Gy of radiation therapy (RT) delivered over 6.5 weeks in 33 fractions with fraction size of $2 \mathrm{~Gy}$ per day from Monday to Friday. A well calibrated course of chemotherapy was given in different settings viz. in neoadjuvant (taxane and cisplatin) and concurrent settings (cisplatin, 5 to 6 courses

Table 1. Demographic distribution of patients of histological proven (squamous cell carcinoma) Head and Neck cancer

\begin{tabular}{lccc}
\hline $\begin{array}{l}\text { Age group } \\
\text { (years) }\end{array}$ & Male & Female & Total \\
\hline $31-40$ & 9 & 2 & 11 \\
$41-50$ & 17 & 3 & 20 \\
$51-60$ & 19 & 1 & 20 \\
$61-70$ & 21 & 1 & 22 \\
$71-80$ & 3 & 1 & 4 \\
Total & $69(89.6 \%)$ & $08(11.3 \%)$ & 77 \\
Mean Age & age $=54.3$ & \\
\hline
\end{tabular}

Table 2. Localization of oral alterations in Head and Neck Cancer and Control

\begin{tabular}{lcccc}
\hline Localization & $\mathrm{N}=77$ & Total (\%) & $\begin{array}{c}\text { Contralateral } \\
\text { Control group }\end{array}$ & Total (\%) \\
\hline Base of tongue & 18 & 23.38 & 9 & 53 \\
Buccal mucosa & 4 & 5.19 & 4 & 23 \\
Pharynx & 4 & 5.19 & Absent & - \\
Upper alveolar region & 4 & 5.19 & Absent & - \\
Larynx & 12 & 15.58 & Absent & - \\
Lateral border of tongue & 2 & 2.5 & 3 & 15 \\
Oropharynx & 23 & 29.87 & Absent & - \\
Soft palate & 7 & 9.09 & Absent & - \\
Throat & 1 & 1.30 & Absent & - \\
Tongue & 2 & 2.60 & 3 & 15 \\
& & & &
\end{tabular}


weekly). Blood counts and blood biochemistry were evaluated before start of chemotherapy with special emphasis on kidney function test (KFT) and general health conditions on Karnofsky performance scale(KPS>70). A combination of chemotherapy and radiation was given as per clinical needs.

The percentage of oral bacterial flora including Staphylococcus aureus was 11.69 in pre RT, which decreased to 5.19 in post-RT, while Escherichia coli was 9.09 in pre RT and increased to 11.69 in post RT. The presence of E.coli was more significant in contralateral control, ( $P$-Value 0.0116) than that in cases. We also found that the percentage of Citrobacter bacteria increased from 10.39 in pre RT to 12.99 in post RT samples. The percentage of $E$. faecalis decreased from 15.58 in pre RT to 6.49 in mid RT. The percentage of $E$. faecium also decreased from 14.29 in pre RT to 7.79 in post RT swab samples, while the percentage of Candida albicans pre RT to post RT was 1.3 - 1.2. And all floras compared with contralateral control and found to be E.coli more than other flora. [Table.3]

Mucositis was a common feature observed in patients undergoing radio- chemotherapy. However, its severity may vary from patient to patient based on radiation dose, type of chemotherapy, and tolerance of individual patients. Mucositis may alter the mucosal surface of the oral cavity and can lead to significant problems during eating, drinking, speaking and during other oral activities. In this study we observed that in $94 \%$ (73/77) of patients, oral mucositis appears at one stage or the other during treatment. As per WHO guidelines for oral mucositis, it was noted that $13.6 \%$ patients showed grade $1(10 / 73)$, and $53 \%$ had grade 2 (39/73) mucositis. Grade 3 mucositis was seen in $31.5 \%(23 / 73)$ and only one patient (1.36\%) presented with grade $4(1 / 73)$ mucositis. Four patients were found to have zero grade mucositis. [Table.4]

Severity of mucositis was correlated with oropharyngeal flora. In grade 0 and grade 1 mucositis, insignificant presence of few organisms was observed while in Grade 2 Citrobacter (33.3\%) E. faecalis (50\%) E. faecium (64\%) E.coli (54\%) Candida albicans (25\%) were found, though the values were insignificant; Staphylococcus aureus was not present in grade 2 mucositis and in grade 3 mucositis. Citrobacter (33.3\%), Staphylococcus

Table 3. Bacterial and yeast type representation in cases and control group

\begin{tabular}{lccccc}
\hline Organism & Pre RT & During RT & Post RT & $\begin{array}{c}\text { Contralateral } \\
\text { Control } \\
\mathrm{N}(\%)\end{array}$ & Significance \\
\hline Staphylococcus Aureus & $9(11.69)$ & $8(10.39)$ & $4(5.19)$ & $2(11.74)$ & $\mathrm{NS}$ \\
Escherichia coli & $7(9.09)$ & $13(16.88)$ & $9(11.69)$ & $6(35.29)$ & $\mathrm{NS}$ \\
Citrobacter & $8(10.39)$ & $9(11.69)$ & $10(12.99)$ & Absent & $\mathrm{NS}$ \\
E. faecalis & $12(15.58)$ & $5(6.49)$ & $12(15.58)$ & Absent & $\mathrm{NS}$ \\
E. faecium & $11(14.29$ & $13(16.80)$ & $6(7.79)$ & $1(5.88)$ & $\mathrm{NS}$ \\
Candida albicans & $1(1.3)$ & $6(7.79)$ & $2(1.2)$ & Absent & $\mathrm{NS}$ \\
\hline
\end{tabular}

* P-value $<0.05$ non significant

aureus (66.6\%) E. faecalis (12.5\%) E. faecium (21\%) E.coli (36\%) Candida albicans (50\%), while in grade 4 mucositis $E$. faecalis was present in only 1 patient. (Table 5 )

When the correlation between oropharyngeal flora and the grade of oral mucositis (severity of acute radiation reaction) was evaluated, a statistically insignificant association was observed between throat swab culture
Table 4. Mucositis grade in patients

Mucositis No. of Patients Percentage Male Female $\begin{array}{ll}\text { Grade } & \text { (N) } 77\end{array}$

\begin{tabular}{lcccc}
\hline Grade 0 & 4 & 5.1 & 3 & 1 \\
Grade 1 & 10 & 13.6 & 9 & 1 \\
Grade 2 & 39 & 53 & 37 & 2 \\
Grade 3 & 23 & 31.5 & 19 & 4 \\
Grade 4 & 1 & 1.36 & 1 & - \\
\hline
\end{tabular}


Table 5. Bacterial and yeast flora present with Mucositis grading

\begin{tabular}{lllllll}
\hline Organisms & $\begin{array}{c}\text { Grade 0 } \\
\mathrm{N}(\%)\end{array}$ & $\begin{array}{c}\text { Grade 1 } \\
\mathrm{N}(\%)\end{array}$ & $\begin{array}{c}\text { Grade 2 } \\
\mathrm{N}(\%)\end{array}$ & $\begin{array}{c}\text { Grade 3 } \\
\mathrm{N}(\%)\end{array}$ & $\begin{array}{c}\text { Grade 4 } \\
\mathrm{N}(\%)\end{array}$ & Significance \\
\hline Staphylococcus aureus & Absent - & $1(33.33)$ & Absent & $2(66.66)$ & Absent & NS \\
E.coli & $1(9.02)$ & Absent & $6(54)$ & $4(36.36)$ & Absent & NS \\
Citrobacter & $2(22.22)$ & $1(11.11)$ & $3(33.33)$ & $3(33.33)$ & Absent & - \\
E. faecalis & Absent & $2(25)$ & $4(50)$ & $1(12.5)$ & $1(12.5)$ & NS \\
E. faecium & Absent & $2(14.2)$ & $9(64.2)$ & $3(21.4)$ & Absent & NS \\
Candida albicans & $1(25)$ & Absent & $1(25)$ & $2(50)$ & Absent & NS \\
\hline
\end{tabular}

status for bacteria and fungi and severity of oral mucositis.

\section{DISCUSSION}

In this study, an attempt has been made to evaluate the oral flora of head and neck cancer patients being treated with a combination of chemotherapy and radiotherapy. The incidence of head and neck cancer was higher in males as compared to females, while the male and female ratio was $8.6: 1$. However, in other studies 7,8 the proportion of males and females was $4: 1$ who were suffering from head and neck cancer. In the present study, three sub-sites in oral cavity were found to be more affected. These were: oropharyngeal region (30\%), base of tongue (23\%), larynx (15.5\%) and soft palate (9\%). Milos Canković et al. ${ }^{9}$ found that most frequent sites of malignancy were floor of the mouth (33.3\%), mandibular gingiva (20\%), and tongue (13\%), while Bhurgri et al. ${ }^{10}$ reported that in oral malignancy, buccal mucosa $(55.9 \%)$ was the most frequently affected site which was followed by the tongue (28.4\%). Another study explained the tongue (67.4\%) as the first frequently affected site, followed by buccal mucosa $(7.7 \%)^{8}$.

Oral mucositis, an acute inflammation of the oral mucosa can be induced by radiotherapy in head and neck cancer patients. Many workers have studied the causes and incidence of oral mucositis. Muthu K et al. ${ }^{11}$ have demonstrated a statistically significant decrease in Alpha Hemolytic Streptococci and Neisseria species post-radiotherapy which resulted in possible recovery from mucositis in head and neck cancer patients However, they also reported a statistically significant increase in Proteus species and Candida albicans in the patients with head and neck cancer post-radiotherapy, while in our study we found an insignificant increase in Candida albicans during RT. The persistence of mucositis is based on multiple factors and oral care and personal hygiene played a significant role. They also found an increase in oropharyngeal colonization with a Gram negative aerobic organism in some cases. The overall mortality in this study was $25.6 \%$. They also observed that gram negative aerobic microorganisms were the cause of death in 7 patients. In our study the mortality rate was $2.5 \%$. Because the maximum patients enrolled in our study were of $2^{\text {nd }}$ and $3^{\text {rd }}$ stage of head and neck cancer, where chances of mortality is less. In some cases the mucosal changes remained even one month after radiotherapy.

Mager DL et al (2005) concluded from their study that the median number of colony forming units (CFUs)/ $\mathrm{ml}$ at the carcinoma site were significantly higher than that at the contralateral healthy mucosa ${ }^{12}$. This may be the possible reason of occurrence of mucositis in patients undergoing chemo-radiotherapy. Wymeng et al (1997) reported that during induction chemotherapy, 5 patients experienced grade II mucositis. One patient was inefficient and 3 were partly dentate, whereas the other patients were fully dentate ${ }^{13}$. While in our study, surprisingly only one patient experienced severe mucositis, while the others suffered from mild to moderate mucositis.

Belazi et al published their findings about their landmark study of mucositis in head and neck cancer patients undergoing radiotherapy. They observed that Candida spp. were present in $77 \%$ patients with oral mucositis ${ }^{14}$. while in our study Candida spp were present in 50\% patients of grade 3 mucositis. This report establishes that bacterial infection and unhygienic conditions of the oral cavity contribute to oral mucositis in patients undergoing radiation therapy. It is prudent 
to say that, monitoring of oral microbes during cancer therapy and initiating appropriate remedial measures can significantly reduce the hardship of patients suffering from mucositis during therapies. In a more recent study, a comparative evaluation was made about presence of microbes in oral cavity in head and neck cancer patients at different stages. This study showed the comparison between oral microbes in patients at the time of diagnosis of oral squamous cell carcinoma (OSCC) with those at mid and end stages of radiotherapy. A statistically significant increase $(P=0.00)$ in Candida spp. was found in patients undergoing radiotherapy ${ }^{15}$. Muthu and his co-workers also found statistically non-significant changes in the buccal mucosa which may be attributed to the altered microbial flora in head and neck cancer patients.

In our study, no statistically significant difference was observed in microbial flora and yeast flora in cases and control group. The oral mucosal samples obtained during preradiotherapy, mid phase of radiotherapy and postradiotherapy in patients with head and neck cancer were compared with control group, though the changes were there but they were not statistically significant. When the grade of mucositis was correlated with presence of bacteria and yeast again, no statistical significance was observed between the two.

\section{CONCLUSION}

All isolated oral flora, except that of $E$. faecalis showed non-significant increase during radiotherapy and decreased post $\mathrm{RT}$, other than Citrobacter which showed an increase while Candida albicans showed a non- significant decrease (83\%) post- radiotherapy.

In radiotherapy, mucositis is firstly the result of direct damage of oral tissue/cells. Patients of head and neck cancer are immunocompromised; hence the oral bacterial colonization prior to radiotherapy predisposes them to mucositis. In patients with head and neck cancer the normal oral bacterial flora are replaced by pathogenic flora during radiotherapy which result in infection. Thus knowledge of altered oropharyngeal bacterial profile in cases of head and neck cancer will help in identifying such infections and devise methods for prevention/treatment.

\section{ACKNOWLEDGMENTS}

None.

\section{CONFLICT OF INTEREST}

The authors declare that there is no conflict of interest.

\section{AUTHORS' CONTRIBUTION}

RM: Data duration, Formal Analysis, Investigation, Methodology, Roles/Writingoriginal draft, Software. MS: Conceptualization, Funding acquisition, Project administration, Supervision, Validation. MR: Investigation, Resources, Visualization. SS: Writing - review \& Editing.

\section{FUNDING}

Funded by the intramural project (IEC No. 28/17) at Dr. RMLIMS, Lucknow.

\section{ETHICS STATEMENT}

This study was conducted in a tertiary cancer care institute, from July 2017 to June 2019. This is an observational analytical case-control study. This Research was dully approved by the Institutional Ethics Committee (IEC No. 28/17).

\section{DATA AVAILABILITY}

All datasets generated or analyzed during this study are included in the manuscript

\section{REFERENCES}

1. Trotti A, Bellm LA, Epstein JB, et al. Mucositis incidence, severity and associated outcomes in patients with head and neck cancer receiving radiotherapy with or without chemotherapy: A systematic literature review. Radiother Oncol. 2003;66(3):253-262. doi: 10.1016/ S0167-8140(02)00404-8

2. Kumar SPS, Balan A, Sankar A, Bose T. Radiation induced oral mucositis. Indian J Palliat Care. 2009;15(2): 95-102. doi: 10.4103/0973-1075.58452

3. Anonymous- Handbook for Reporting Results of Cancer Treatment. Vol. 48. WHO Offset Publication; 1979:1522.

4. Redding SW, Zellars RC, Kirkpatrick WR et al. Epidemiology of oropharyngeal Candida colonization and infection in patients receiving radiation for oral cancer. J Clin Microbiol. 1999:37(12):3896-900 doi: 10.1128/JCM.37.12.3896-3900.1999

5. Bagg J, Sweeney MP, Lewis MAO, et al. High prevalence of non-albicans yeasts and detection of anti-fungal resistance in the oral flora of patients with advanced cancer. Palliat Med. 2003;17:477-81. doi: 10.1191/0269216303pm793oa 
6.

Belazi M, Velegraki A, Koussidou ET, et al. Oral Candida isolates in patients undergoing radiotherapy for oral cancer. prevalence, azole susceptibility profiles and response to antifungal treatment. Oral Microbiol Immunol. 2004;19(6):347-355. doi: 10.1111/j.1399302x.2004.00165.x

7. Mehrotra R, Pandya S, Chaudhary AK, Kumar M, Singh $M$. Prevalence of oral premalignant and malignant lesions at a tertiary level hospital in Allahabad, India. Asian Pac J Cancer P. 2008;9:263-66.

8. Iype EM, Pandey M, Mathew A, Thomas G, Sebastian $P$, Nair MK. Oral cancer among patients under the age of 35 years. J Postgrad Med. 2001;47:171-176.

9. Milos C, Marija BB, Jovana L, Jovan M, Miroslav PI. Bacterial flora on the surface of oral squamous cell carcinoma. Arch Oncol. 2013;21(2):62-64. doi: 10.2298/AOO1302062C

10. Bhurgri Y. Cancer of the oral cavity-trends in Karachi South (1995-2002). Asian Pac J Cancer P. 2005;6(1):2226.

11. Muthu K, Raman R, Gopalakrishnan G. Oropharyngeal flora changes in patients with head and neck malignancy post radiotherapy. Med J Malaysia.
2004;59(5):585-90.

12. Mager DL, Haffajee AD, Devlin PM, Norris CM, Posner MR, Goodson JM. The salivary microbiota as a diagnostic indicator of oral cancer: A descriptive, nonrandomized study of cancer-free and oral squamous cell carcinoma subjects. J Transl Med. 2005;3:27. doi: 10.1186/1479-5876-3-27

13. Wymenga A, Graaf WTA, Spijkervet FLK, et al. A new in-vitro assay for qualitative of chemotherapy induced mucositis. Br J Cancer. 1997;76:1062-1066. doi: 10.1038/bjc.1997.508

14. Belazi M, Velegraki A, Koussidou-Eremondi T, et al. Oral Candida isolates in patients undergoing radiotherapy for oral cancer: prevalence, azole susceptibility profiles and response to antifungal treatment. Oral Microbiol Immunol. 2004;19(6):347-51. doi: 10.1111/j.1399302x.2004.00165.x

15. Sonalika WG, Amsavardani TS, Bhat KG, Patil BR, Muddapur MV. Oral microbial carriage in oral squamous cell carcinoma patients at the time of diagnosis and during radiotherapy - a comparative study. Oral Oncol. 2012;48(9):881-886. doi: 10.1016/j. oraloncology.2012.03.018 